\title{
Real-Time Diagnostics at ASDEX Upgrade- Integration with MHD Feedback Control
}

W. Treutterer, K. Behler, L. Giannone, N. Hicks, A. Manini, M. Maraschek, G. Raupp, M. Reich, A.C.C. Sips, J. Stober, W. Suttrop and ASDEX Upgrade Team

Max-Planck Institut für Plasmaphysik, Boltzmannstraße 2, D-85748 Garching, Germany, EURATOM Association

\begin{abstract}
At the ASDEX Upgrade tokamak experiment, a new feedback control loop is under construction with the aim of stabilizing magneto-hydrodynamic (MHD) instabilities, such as neoclassical tearing modes and sawteeth. It uses the mirrors of the electron cyclotron heating $(\mathrm{ECH})$ launchers, which can be steered in real-time to guide each beam to the position needed to stabilize and suppress the mode.
\end{abstract}

The control system needs highly specialized plasma state information such as island position and ECH beam deposition locations in real-time. Data from several diagnostic systems, like electron cyclotron emission (ECE), magnetic measurements and motional Stark effect must be combined in real-time to obtain the required information. These systems strongly differ in sampling characteristics and time resolutions. High sampling rates as $2 \mathrm{MHz}$ for ECE are often required to provide enough data for correlation or frequency analysis. On the other hand, complex analysis methods, such as equilibrium and profile reconstruction, may operate on slower rates of some milliseconds and need tight interaction with measurement systems and high computing power.

In this paper, we describe a concept for distributed real-time diagnostic data handling, integration of data from several asynchronous diagnostic systems, and connection to the discharge control system for a broad spectrum of requirements. The system is structured into distributed diagnostic computer clusters, a realtime signal server to combine all information, and the discharge control system. While the focus is currently on MHD control, further real-time diagnostic related applications will be added in future.

Keywords : Real-time diagnostic, control system, MHD stabilization, ECH 


\section{Introduction}

The impact of magneto-hydrodynamic (MHD) instabilities in the plasma core is so important that sufficient performance can only be achieved, if these instabilities are actively controlled. Overviews of the stabilization of MHD modes and the feedback concept planned for ASDEX Upgrade are described in [1] and [2] respectively. In summary, the presence of a mode in the plasma flattens the temperature profile, which is measured by the electron cyclotron emission (ECE). It produces fluctuations in the toroidal symmetry of the poloidal magnetic field, which can be detected by Mirnov coils. Further magnetic flux and field measurements, together with polarization angles measured using the motional Stark effect (MSE), allow to reconstruct the internal magnetic plasma structure and to map all information to a common coordinate system. From these raw measurements one can extract the physical quantities of interest: the mode 's location, size and amplitude. Figure 1 shows, how the magnetic flux surfaces link island position, ECE observation channels and ECH beam deposition and illustrates the necessity of combined diagnostic evaluation. With this information the controller directs the beams of the electron cyclotron heating $(\mathrm{ECH})$ to the resonant flux surface, where the mode is located, by moving the steerable mirrors [3]. There, by depositing power and driving current, the mode is stabilized. Figure 2 shows the resulting control loop. MHD control is supposed to become a permanently available tool also in standard plasma operation and is planned also for ITER.

All methods require the combined evaluation of several multi-channel diagnostic systems, executing analysis algorithms of considerable complexity and a prompt and accurate reaction of the actuator, computed by the discharge control system (DCS) [4]. The key challenge is to integrate diagnostic systems with the control system in real-time. The basic idea - sharing diagnostic data over a real-time data network - is common to many other fusion devices like DIII-D [5], JET [6] and JT60 [7]. The details, however, vary according to the control system philosophy and networking hardware. Our approach comprises several steps: adding real-time capabilities and preprocessing algorithms to diagnostic systems (section 2), defining a software framework for integration of diagnostic data from asynchronous sources (section 3), implementing this framework on a real-time signal server with connection to the discharge control system (section 4), and finally designing a controller to generate commands for the ECH actuator system (section 5). 


\section{Transition to real-time diagnostics}

Previously, measured data were just recorded and analysed off-line with no time limitations for signal conditioning, filtering, and computational algorithms. Information from other diagnostic systems were readily available and could easily be mapped to a common time base. The real-time constraint makes all those steps considerably harder.

An example is the data acquisition process. For off-line analysis it was most efficient to transfer the whole measurement data all-in-one from an external sampling device into the collecting computer's memory for processing and archiving. But for real-time control, data transfer has to be broken down into many small packets, which must be processed in-time, to feed the controller. Algorithms must also obey the causality constraint: only previously sampled data can be processed. Future samples, which could help in phase-preserving filtering, averaging or curve fitting interpolation, are not available.

On the other hand data conditioning and reduction is indispensable for further processing in order to reduce noise, provide meaningful values and comply with the communication network bandwidth and computing power available. MSE, magnetic, ECE, and Mirnov diagnostic systems each acquire between 10 and 100 channels at sampling rates from $100 \mathrm{kHz}$ to $2 \mathrm{MHz}$. For magnetic probe processing and equilibrium calculation we use a high-performance LabView installation [8]. The other diagnostic systems typically comprise several multi-core computers with real-time enabled Solaris or Linux operating system, which can be tightly coupled in network clusters. Their combined computing power is used to condition measurements with filtering, correlation and frequency analysis algorithms. These algorithms are static components of the diagnostic. Using the message passing interface (MPI) library provides a software layer for efficient parallel computation of the respective algorithms on distributed nodes [9]. The results, magnetic flux distribution, ECE channel correlation profiles and mode amplitudes typically come at data rates of $100 \mathrm{~Hz}-1 \mathrm{kHz}$.

\section{Diagnostic data integration}

To obtain physically meaningful parameters, however, diagnostic data must often be further condensed. The ECE correlation profiles describe the mode location in ECE channel space. To become comparable with the ECH beam deposition location, a mapping to the plasma's magnetic flux surface structure is 
necessary, yielding the normalized radii $\rho_{\text {res }}$ and $\rho_{\text {dep }}$ or even their projections on the ECH launcher mirror drive positions. For this we need input from magnetic probes and MSE polarization angles. With the availability of more real-time diagnostic systems like Thomson scattering combined with real-time beam tracing and Soft X-ray radiation, these values can be calculated also in alternative ways and Bayesian filters will be applied to extract the best estimation.

This is the point, where information from several diagnostic systems has to be combined, and here the problem of synchronising the data arises. A common sampling and evaluation time grid would simplify the case significantly but cannot generally be imposed. Due to the type and amount of data, measurement principle and technical considerations, each diagnostic system may use its own sampling rate and triggering onset. Many algorithms, on the other hand, assume that their input data represent a snapshot of a plasma state and have been captured simultaneously. In order to avoid side-effects from jitter, asynchronous input data must be interpolated before use. To accomplish synchronisation, all data, raw measurements as well as processed results, must be tagged with an appropriate time marker.

Additionally, a status tag should indicate whether the value is valid and when production has stalled. MSE data, for example, is valid only during neutral beam injection. Subsequent algorithms must be aware of these limitations.

Some algorithms may also behave differently in certain plasma regimes. For transparent operation it is essential to be able to review these events and internal decisions later on. Thus, a message logging facility like in classical control systems is needed.

\section{Real-time signal data server}

The data exchange between diagnostic systems and the discharge control system is deployed on a selfcontained instance, the real-time signal server (RTSS). Figure 3 illustrates the role of the RTSS in the control loop. This server will initially be implemented as one computation node, but may be extended to a computer cluster later on. The diagnostic systems provide their condensed, time-stamped data, together with a status tag, as "signals" to the server. Starting with a socket based network protocol, we aim at the use of Infiniband technology later.

The RTSS has three functions. First, it moderates data exchange among the clients - real-time diagnostic 
systems and the discharge control system - providing synchronisation and interpolation services as well as transparent signal routing keeping signal producers independent from signal consumers. Thus, it is straightforward to add new features without touching existing code. Second, it hosts high-level data processing algorithms integrating inputs from several client systems. Finally, it allows for dedicated hardand software implementations of the clients. The real-time signal network performance of DCS, for instance, optimized for many small data packets and $1 \mathrm{kHz}$ base cycle at minimum latency would considerably degraded, if it was used to transfer the big packets coming from the ECE and magnetic diagnostic systems.

On the other hand, the RTSS inherits proven key concepts from the DCS software infrastructure framework like extensibility with plug-in modules, signal routing, message logging, and parameter configuration via a central parameter server. Due to this common ground, the RTSS and DCS might be combined in future, when the evolution of real-time operating systems and high-bandwidth network solutions allows it.

\section{MHD controller}

The reconstructed physical parameters on the RTSS finally feed the MHD controller, implemented as part of the DCS, which has access to all the experiment's actuators. It generates proper output commands for $\mathrm{ECH}$ mirror drives and gyrotron powers to heat the plasma and drive current at the correct location and time. The latency, from measurement capturing to command execution, must be short enough in order not to hit the wrong position in which case the control loop could become unstable. Given the MHD growth rate $(\sim 100 \mathrm{~ms})$, the control cycle and hence the update rate of reconstruction algorithms should be $10 \mathrm{~ms}$ or faster to achieve good results.

\section{Summary}

In this paper we described an approach, to integrate real-time capable diagnostic systems with the discharge control system. Data flow optimization and software infrastructure services for synchronisation, data routing and message logging are the key elements of this approach. The generic approach allows extension to other applications like plasma density and pressure profile reconstruction or disruption 
precursor calculation. The MHD control system is currently under construction. First results are expected by end 2007 . 


\section{References:}

[1] H. Zohm, et.al., Control of MHD instabilities by ECCD: AUG results and implications for ITER, Nuclear Fusion 47 (2007) 228

[2] A. Manini, et.al., Development of a feedback system to control MHD instabilities in ASDEX Upgrade, $24^{\text {th }}$ SOFT (2006), Warsaw, to be published in Fus. Eng. and Design

[3] F. Leuterer, et. al., Status of the New ECRH System for ASDEX Upgrade, Fus. Eng. and Design, Vol. 74 (2005), $199-203$

[4] G. Raupp, et. al., Control process structure of ASDEX Upgrade's new Control and Data Acquisition System, Fus. Eng. and Design, Vol. 74 (2005), 697-705.

[5] B.G. Penaflor, et.al., Current status of the upgraded DIII-D real-time digital plasma control system, Fusion Eng. and Design, Vol. 71 (2004), $47-52$.

[6] R. Felton, et.al., Real-time measurement and control at JET experiment control, Fusion Eng. and Design, Vol. 74 (2005), $561-566$.

[7] K. Kurihara, et, al., Status and prospect of JT-60 plasma control and diagnostic data processing systems for advanced operation scenarios, Fusion Eng. and Design, Vol. 81 (2006), 1729 - 1734.

[8] L. Giannone, et. al., Real time magnetic flux surface positions for ASDEX Upgrade, Proc. $34^{\text {th }}$ EPS on Plasma Physics (2007), Warsaw

[9] K. Behler, et. al., Real-Time Diagnostics at ASDEX Upgrade - Architecture and Operation, this conference 


\section{Figures:}

Figure 1: MHD control principle

Figure 2: Information flow for MHD control

Figure 3: Integration of real-time diagnostics in the control loop 